\title{
Les opticiens de l'Alberta possèdent-ils les compétences requises pour exécuter des examens de la réfraction et prescrire un appareil optique de façon autonome et en toute sécurité?
}

\author{
Alyssa Anderson, B. Sc., \\ candidate à la maîtrise \\ en politiques publiques \\ Collège des optométristes \\ de l'Alberta \\ Gordon Hensel, OD, FAAO \\ Collège des optométristes \\ de l'Alberta
}

\begin{abstract}
Résumé
L'Alberta est l'une des trois provinces canadiennes qui autorisent les opticiens à effectuer des examens de la réfraction. Récemment, l'Alberta College and Association of Opticians (ACAO) a demandé que le champ d'exercice des opticiens soit élargi pour inclure la capacité de prescrire des appareils optiques, qui est actuellement réservée aux optométristes et aux ophtalmologistes. Tout au long du présent document, le terme « prescrire » devrait être interprété comme la prescription d'un appareil optique, et non comme celle d'un agent pharmaceutique thérapeutique. Dans le présent document, nous cherchons à déterminer si les opticiens qui effectuent des examens de la réfraction désignés en Alberta ont une formation et des connaissances adéquates pour exécuter un examen et prescrire un appareil optique de façon autonome en toute sécurité. Pour répondre à cette question de recherche, nous avons dressé une liste de 27 compétences qui selon nous sont requises pour prescrire et faire des examens de la réfraction de façon autonome en toute sécurité. Nous avons ensuite évalué les programmes d'optique du Northern Alberta Institute of Technology (NAIT) et les examens d'entrée dans la profession administrés par l'Association nationale des autorités de réglementation des opticiens du Canada (NACOR) de même que le Bureau des examinateurs en optométrie du Canada (BEOC) pour déterminer s'ils couvrent ces 27 compétences. Nos constatations montrent que les programmes de sciences optiques du NAIT ne couvrent pas ou n'évaluent pas dans la pratique plus de la moitié ( $59 \%$ ) des compétences requises pour prescrire et exécuter des examens de la réfraction de façon autonome en toute sécurité, et que l'examen de la NACOR ne couvre pas $77 \%$ de ces compétences. Selon cette information, les opticiens qui effectuent des examens de la réfraction désignés en Alberta ne possèdent pas la formation et les connaissances nécessaires pour exécuter cet examen et prescrire un appareil optique de façon autonome en toute sécurité. Le fait d'accorder aux opticiens le pouvoir légal de prescrire et d'exécuter des examens de façon autonome peut donner lieu à un problème de santé publique, car il pourrait y avoir une augmentation du nombre de maladies oculaires et systémiques non diagnostiquées ou non détectées.
\end{abstract}

MOTS CLÉS :

Alberta, opticien, optométriste, examen de la réfraction (EdR), prescrire, formation 


\section{INTRODUCTION}

Les opticiens sont des professionnels des soins oculovisuels formés pour concevoir, ajuster, et distribuer des appareils optiques sur ordonnance d'un optométriste ou d'un médecin ${ }^{1}$. L'Alberta et deux autres provinces canadiennes (la Colombie-Britannique et l'Ontario) ont accordé aux opticiens ayant une certification spéciale le pouvoir législatif de faire des examens de la réfraction. Toutefois, aucune administration ne permet actuellement aux opticiens de prescrire des appareils optiques.

Dans ce document, nous avons cherché à déterminer si les opticiens qui effectuent des examens de la réfraction en Alberta ont la formation et les connaissances nécessaires pour exécuter un EdR/P de façon autonome en toute sécurité. Pour effectuer un EdR/P en toute sécurité, un professionnel des soins oculovisuels doit posséder les compétences et les connaissances nécessaires pour accomplir la tâche avec compétence sans causer de blessure ou de préjudice au patient. Cette question de recherche est importante parce que l'Alberta College and Association of Opticians (ACAO) a demandé que son champ d'exercice soit élargi pour inclure le pouvoir législatif de prescrire². En Alberta, la prescription est considérée comme une activité restreinte ${ }^{3}$. Pour s'assurer que le public est protégé de façon appropriée, seul un professionnel de la santé réglementé ayant les compétences, les habiletés et les connaissances appropriées peut effectuer une activité restreinte précise. Étant donné que les optométristes se sont déjà vu accorder l'activité restreinte de prescription, il pourrait être utile de comparer les connaissances et les compétences des opticiens en matière d'EdR/P à celles des optométristes. Cette comparaison aidera à déterminer si les opticiens qui réalisent des examens de la réfraction de l’Alberta possèdent un ensemble de compétences appropriées pour effectuer cet examen et prescrire de façon autonome en toute sécurité sans exposer le public à un risque indu.

MÉTHODES :

Pour répondre à cette question de recherche, nous avons utilisé la démarche suivante :

1) Compilation d'une liste des compétences clés jugées nécessaires pour exécuter un examen de la réfraction et prescrire un appareil optique de façon autonome en toute sécurité

2) Évaluation des programmes de sciences optiques du Northern Alberta Institute of Technology (NAIT) en ce qui a trait à la couverture de ces compétences

3) Comparaison de la couverture de ces compétences par les examens d'admission à la pratique administrés par l'Association nationale des autorités de réglementation des opticiens (NACOR) et le Bureau d'examen en optométrie du Canada (BEOC)

Ce document décrit les résultats de chacune de ces étapes, tire des conclusions et formule des recommandations quant à la question de savoir si les opticiens de l'Alberta qui exécutent des EdR possèdent les compétences et les connaissances adéquates pour réaliser cet examen et prescrire un appareil optique de façon autonome en toute sécurité.

COMPÉTENCES CLÉS REQUISES POUR EFFECTUER UN EDR ET PRESCRIRE DE FAÇON AUTONOME

Pour exécuter un EdR/P de façon sécuritaire, un professionnel des soins oculovisuels doit examiner ou évaluer cinq aspects $^{4}$ :

- activités professionnelles et de loisirs et exigences connexes;

- état de santé systémique;

- état de réfraction et acuités visuelles;

- état de la vision binoculaire;

- état de santé oculaire.

Il faut noter que l'exécution d'un EdR et la prescription d'un appareil optique de façon autonome en toute sécurité exigent plus que la simple capacité de déterminer l'état de réfraction et l'acuité visuelle d'un patient. Si des facteurs comme l'état de santé oculaire ou systémique, l'état de la vision binoculaire ou des considérations professionnelles ou de loisirs ne sont pas pris en compte, le professionnel des soins oculovisuels peut exposer le patient à la progression de maladies oculaires non diagnostiquées ou à des effets secondaires d'une ordonnance inappropriée (maux de tête ou fatigue oculaire). Par conséquent, l'exécution d'un EdR/P de façon autonome en toute sécurité exigent des compétences et des capacités qui dépassent la capacité d'exécuter l'examen lui-même. Le tableau 1 présente les 27 compétences que nous avons jugées nécessaires pour exécuter un EdR/P de façon autonome en toute sécurité selon les cinq domaines énumérés ci-dessus. 
Un professionnel des soins oculovisuels qui effectue des examens de réfraction et émet des ordonnances sans être en mesure d'exécuter l'ensemble de ces 27 techniques peut poser un risque important pour la santé publique. Ensuite, ces 27 compétences ont été comparées à 1) les compétences enseignées dans les trois programmes de sciences optiques du NAIT et 2) les compétences évaluées dans les examens d'admission à la pratique de la NACOR et du BEOC.

ÉVALUATION DES PROGRAMMES DE SCIENCES OPTIQUES DU NAIT

Le NAIT est l'un des sept établissements d'enseignement au Canada qui offrent un programme de formation en techniques d'orthèses visuelles accrédité par la NACOR (tableau 2)5.

Tableau 1 : Compétences clés requises pour effectuer un EdR et prescrire

\begin{tabular}{|c|c|}
\hline Domaine & Compétences requises \\
\hline $\begin{array}{l}\text { État de réfraction et acuité } \\
\text { visuelle }\end{array}$ & $\begin{array}{l}\text { - Effectuer un EdR objective à l'aide d'un rétinoscope ou d'un autoréfractomètre; } \\
\text { - Effectuer un EdR subjective à l'aide d'un phoroptère ou d'un ensemble de lentilles; } \\
\text { - Utiliser les échelles d'acuité à distance et à proximité } \\
\text { - Utiliser un kératomètre pour mesurer la courbure cornéenne } \\
\text { - Mesurer la puissance de la lentille à l'aide d'un lensomètre }\end{array}$ \\
\hline État de santé oculaire & $\begin{array}{l}\text { - Effectuer un examen des segments antérieur et postérieur } \\
\text { - Utiliser des appareils de photographie des segments antérieurs et postérieurs } \\
\text { - Utiliser un instrument à balayage laser } \\
\text { - Effectuer une tonométrie à l'aide d'un tonomètre } \\
\text { - Effectuer un test informatisé du champ visuel } \\
\text { - Effectuer un test de vision des couleurs } \\
\text { - Utiliser une lampe à fente } \\
\text { - Utiliser un ophtalmoscope } \\
\text { - Utiliser une lampe-stylo } \\
\text { - Utiliser un pachymètre }\end{array}$ \\
\hline État de santé systémique & $\begin{array}{l}\text { - Capacité de détecter des signes de maladie systémique dans l'œil et ses annexes } \\
\text { - Capacité de demander des tests de laboratoire } \\
\text { - Utiliser un exophthalmomètre } \\
\text { - Utiliser un sphygmomanomètre }\end{array}$ \\
\hline $\begin{array}{l}\text { État de la vision } \\
\text { binoculaire }\end{array}$ & $\begin{array}{l}\text { - Effectuer des tests pour évaluer l'état de la vision binoculaire } \\
\text { - Effectuer un test pour évaluer la fonction d'accommodation } \\
\text { - Effectuer un test pour évaluer la motilité oculaire } \\
\text { - Effectuer un test pour évaluer la dominance oculaire } \\
\text { - Effectuer des tests pour évaluer la concomitance oculaire } \\
\text { - Effectuer des tests pour détecter des désordres sensoriels et d'autres problèmes de perception }\end{array}$ \\
\hline $\begin{array}{l}\text { Activités professionnelles } \\
\text { et de loisirs }\end{array}$ & $\begin{array}{l}\text { - Capacité à comprendre les besoins des patients } \\
\text { - Capacité à communiquer les options appropriées au patient }\end{array}$ \\
\hline
\end{tabular}

Tableau 2 : Programmes d'optique accrédités par l l’Association nationale des autorités de réglementation des opticiens

\begin{tabular}{|l|l|}
\hline Établissement d'enseignement/programme & Lieu \\
\hline Programme de sciences optiques du NAIT & Edmonton, Alberta \\
\hline Seneca College Opticianry Program & Toronto, Ontario \\
\hline Douglas College Dispensing Optician Program & New Westminster, Colombie-Britannique \\
\hline Oulton College Optician Diploma Program & Moncton, Nouveau-Brunswick \\
\hline $\begin{array}{l}\text { Programme Techniques de lunetterie et de contactologie du Collège } \\
\text { communautaire du Nouveau-Brunswick }\end{array}$ & Moncton, Nouveau-Brunswick \\
\hline Stenberg College Dispensing Optician Program & Surrey, Colombie-Britannique \\
\hline Programme d'optique du Georgian College & Barrie, Ontario \\
\hline
\end{tabular}

Trois collèges du Québec offrent aussi des programmes de techniques d'orthèses visuelles, mais ces programmes ne sont pas accrédités par la NACOR ${ }^{16}$. 
Le programme de sciences optiques du NAIT est unique en ce sens qu'il offre un système en trois étapes pour l'étude de l'optique. Dans le système du NAIT, les étudiants commencent par obtenir un diplôme en ajustement de lunettes ${ }^{6}$. Par la suite, les étudiants peuvent obtenir une formation spécialisée en obtenant un certificat en lentilles cornéennes et un certificat en réfraction ${ }^{78}$. Les étudiants des programmes du NAIT doivent travailler dans le domaine de l'optique avec un opticien autorisé, un optométriste ou un ophtalmologiste qui supervisera le volet pratique de chaque programme.

Nous avons utilisé les plans de cours accessibles au public du NAIT pour déterminer si les 27 compétences requises pour prescrire et exécuter des examens de la réfraction en toute sécurité sont couvertes dans les trois programmes de sciences optiques. Les programmes de sciences optiques du NAIT couvrent et évaluent en pratique 11 (40,7\%) des 27 compétences que nous avons jugées nécessaires pour exécuter des examens de la réfraction et prescrire en toute sécurité. Cinq (18,5\%) sont couvertes, mais sans évaluation pratique, et 11 (40,7 \%) ne sont pas couvertes (figure 1). Par conséquent, les programmes de sciences optiques du NAIT ne couvrent pas ou n'évaluent pas en pratique plus de la moitié (59,2\%) des compétences requises pour exécuter des examens de la réfraction et prescrire en toute sécurité. Ce qui est peut-être le plus important, c'est que les programmes du NAIT couvrent et évaluent en pratique seulement $20 \%$ des compétences requises pour évaluer la santé oculaire (2/10) et ne voient aucune des compétences requises pour évaluer la santé systémique (0/4), tout en couvrant et évaluant concrètement $80 \%$ des compétences requises pour évaluer l'état de réfraction (4/5).

Figure 1 : Proportion des compétences liées à l'EdR et à la prescription couvertes par les programmes de sciences optiques du NAIT.

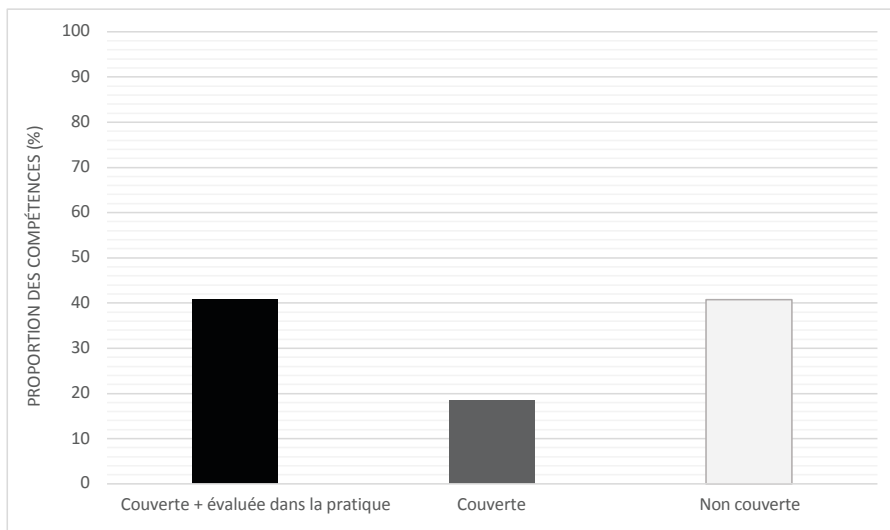

La figure 2 montre une répartition de la couverture des compétences par le NAIT en fonction des cinq domaines requis pour exécuter des examens de la réfraction et prescrire en toute sécurité.

Figure 2 : Proportion des compétences dans les cinq domaines nécessaires à l'EdR et à la prescription sécuritaires couvertes par le NAIT. Consultez l'annexe A pour obtenir une liste des compétences particulières qui sont couvertes, non couvertes et évaluées dans la pratique dans les programmes du NAIT.

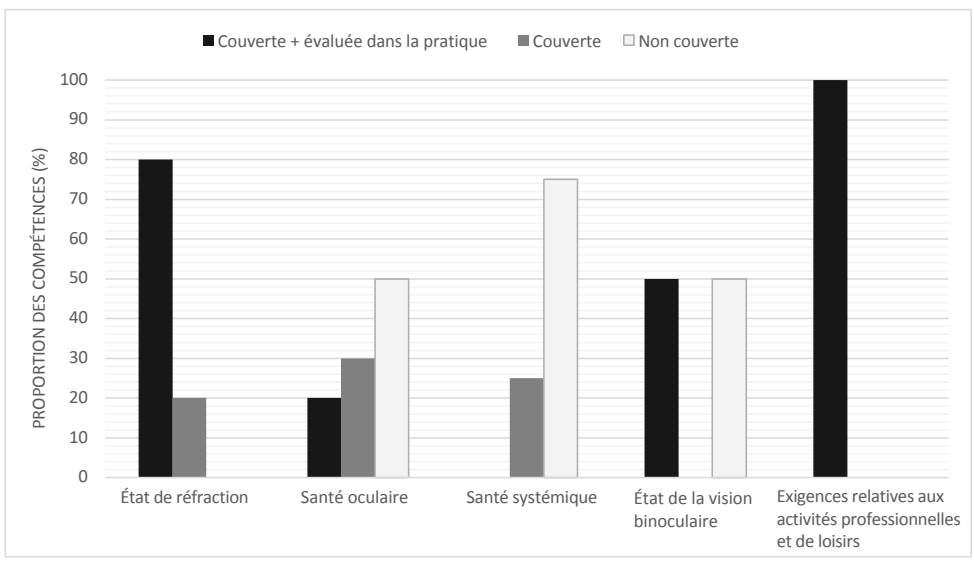


EXAMEN DE LA NACOR PAR RAPPORT À L’EXAMEN DU BEOC

La NACOR est un organisme de réglementation indépendant qui administre les examens d'entrée dans l'exercice de la profession pour les opticiens 9 . La NACOR administre actuellement deux examens, soit l'examen Optical Science 1 (lunettes) et l'examen de pratique avancée Optical Science 2 (lentilles cornéennes).

Le BEOC est un organisme sans but lucratif qui administre l'examen d'entrée dans l'exercice de la profession en optométrie ${ }^{10}$.

Les examens de la NACOR couvrent 22,2 \% des compétences jugées nécessaires pour exécuter des examens de la réfraction et prescrire un appareil optique de façon autonome en toute sécurité (6/27 compétences), tandis que l'examen du BEOC couvre 85,2 \% de ces compétences (23/27 compétences) (figure 3$)^{11,12}$.

Figure 3 : Proportion des compétences liées à l'EdR et à la prescription couvertes dans les examens de la NACOR et du BEOC. Consultez l'annexe B pour obtenir une liste des compétences particulières qui sont couvertes par les examens de la NACOR et du BEOC.

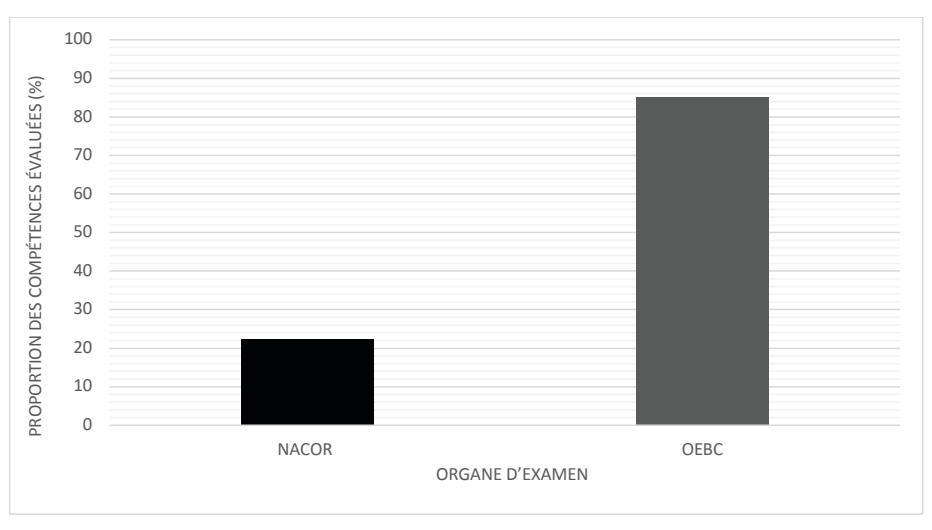

\section{DISCUSSION}

Les opticiens de l'Alberta qui exécutent des examens de la réfraction sont formés pour effectuer ces examens en suivant les trois programmes d'optique du NAIT, qui ne couvrent pas ou n'évaluent pas en pratique les élèves sur plus de la moitié (59,2 \%) des compétences que nous avons jugées nécessaires pour exécuter des examens de la réfraction et prescrire de façon autonome en toute sécurité (figure 1). Plus important encore, les programmes du NAIT ne couvrent pas de manière adéquate et n'évaluent pas de manière pratique ce qui est sans doute le plus important des cinq domaines que nous considérons comme nécessaires pour exécuter des examens de la réfraction et prescrire en toute sécurité un appareil optique (la santé oculaire et la santé systémique d'un individu), couvrant respectivement $20 \%$ et $0 \%$ des compétences requises, tout en couvrant et en évaluant de manière pratique $80 \%$ des compétences requises pour évaluer l'état de réfraction (4/5) (Figure 2). Cela donne à penser que les programmes de science optique du NAIT permettent aux opticiens de l'Alberta d'acquérir les compétences nécessaires pour effectuer un EdR simple, mais ne couvrent pas adéquatement et n'évaluent pas de façon pratique les compétences qui seraient nécessaires pour rédiger une ordonnance complète. Pour cette raison, nous concluons que la formation offerte dans les trois programmes de sciences optiques du NAIT n'est pas adéquate pour permettre aux opticiens d'exécuter des examens de la réfraction et de prescrire un appareil optique de façon autonome en toute sécurité. Toutefois, étant donné que les programmes couvrent et évaluent dans la pratique quatre des cinq techniques nécessaires pour évaluer l'état de réfraction d'un patient, la formation peut être suffisante pour permettre aux opticiens qui exécutent des EdR d'évaluer l'état de réfraction comme composante d'un examen complet de la vue effectué par un optométriste ou un ophtalmologiste.

Nous avons aussi constaté que les examens de la NACOR n'évaluent pas les candidats sur 77,8 \% des compétences requises pour prescrire et exécuter des examens de la réfraction en toute sécurité (figure 3), y compris la capacité d'effectuer des examens objectifs et subjectifs, des tests de vision binoculaire, et des examens de la santé oculaire des segments antérieur et postérieur. Par conséquent, d'après le contenu des examens de la NACOR, les connaissances et les compétences requises pour exécuter un EdR/P de façon autonome en toute sécurité des opticiens de l'Alberta qui exécutent ces examens ne sont pas adéquatement évaluées. 
D'après notre analyse du contenu couvert dans les programmes de sciences optiques du NAIT et le contenu de l'examen de la NACOR, nous concluons que les opticiens de l'Alberta qui exécutent des EdR ne possèdent pas la formation et les connaissances adéquates pour exécuter ces examens et prescrire un appareil optique de façon autonome en toute sécurité. Nos constatations font ressortir la possibilité d'un problème de santé publique. Une étude menée en 2014 a révélé que plus de $25 \%$ des patients qui se présentaient dans un cabinet d'optométriste avec des symptômes uniquement liés à la réfraction ont reçu un diagnostic de problème oculovisuel asymptomatique ${ }^{13}$. Cette étude fait ressortir la possibilité d'un problème de santé publique, car les problèmes de santé oculaire des patients peuvent passer inaperçus et ne pas être diagnostiqués par les opticiens qui exécutent les examens de la réfraction. Le vieillissement de la population de l'Alberta pourrait aussi accroître le nombre de problèmes oculaires qui pourraient être négligés par les opticiens qui exécutent les examens de la réfraction. Les données obtenues des Services de santé de l'Alberta (annexe C) indiquent que, entre les exercices 2013-2014 et 2015-2016, il y a eu au total 487066 diagnostics liés aux yeux en Alberta $^{14}$. De plus, au cours de l'exercice 2014-2015, le gouvernement de l'Alberta a dépensé environ 239,6 millions de dollars pour le traitement des maladies liées aux yeux et des troubles connexes. L'exécution d'examens de la réfraction (et encore moins des prescriptions) sans évaluation/examen de la santé oculaire et systémique en Alberta peut accroitre le risque qu'une maladie oculaire ou systémique ne soit pas diagnostiquée ou dépistée. En retour, cela pourrait entraîner une incidence plus élevée de perte de vision évitable et une augmentation des dépenses gouvernementales en soins oculovisuels. Par conséquent, du point de vue de la santé publique, il est impératif de ne pas accorder aux opticiens le pouvoir législatif d'exécuter un EdR et de prescrire de façon autonome un appareil optique en raison de leur manque de connaissances sur la santé oculaire et la vision binoculaire.

Étant donné que le gouvernement de l'Alberta a déjà accordé aux opticiens spécialement formés le droit d'exécuter des EdR de façon autonome, et à la lumière des limites importantes identifiées dans la formation et l'évaluation actuelles des opticiens, nous recommandons que NACOR développe un troisième examen d'entrée dans la pratique de la profession conçu pour les opticiens cherchant à acquérir la capacité d'exécuter des examens de la réfraction de façon autonome. L'examen ne devrait être ouvert qu'aux opticiens des provinces qui autorisent les examens de la réfraction réalisés par les opticiens (Alberta, C.-B. et Ontario). De plus, la NACOR devrait travailler en collaboration avec le BEOC afin de dresser une liste de compétences et de mettre en place des processus d'examen appropriés; quand la loi confère aux opticiens et aux optométristes le droit d'exécuter des examens de la réfraction, les compétences des candidats en matière d'EdR dans les deux domaines devraient être évaluées de la même façon. En élaborant un examen propre aux opticiens qui exécutent des examens de la réfraction, la NACOR peut s'assurer que les opticiens possèdent un ensemble de compétences plus approprié pour accomplir les tâches liées à cet examen qui relève actuellement de leur champ d'exercice.

Soit dit en passant, il faut noter que l'examen du BEOC n'a pas permis d'évaluer adéquatement quatre des 27 compétences liées à l'EdR et à la prescription. Nous recommandons donc que le BEOC modifie son examen pour couvrir toutes les compétences décrites dans le présent document.

Nous recommandons également que le NAIT modifie la partie clinique de son certificat de réfraction. À l'heure actuelle, le volet clinique du certificat de réfraction peut être supervisé par un opticien autorisé, un technologue médical en ophtalmologie, un optométriste ou un ophtalmologiste ${ }^{15}$. Cette différence en matière de supervision pourrait entraîner une variabilité des résultats d'apprentissage des étudiants. Par conséquent, il est recommandé que le NAIT normalise la description de tâche des superviseurs clinique de façon à ce que ce soit les personnes qui sont le plus susceptibles de posséder une majorité importante des compétences en question qui aient cette responsabilité. Grâce à leur vaste formation clinique, à leur vaste champ d'exercice et à leur expérience des examens de la réfraction, ce sont les optométristes autorisés/inscrits qui répondent le mieux à ce critère, ce qui devrait réduire le risque de variabilité des résultats d'apprentissage des étudiants.

Nos méthodes pourraient être limitées par un certain nombre de facteurs. Premièrement, les plans de cours du NAIT et les examens types de la NACOR et du BEOC donnent simplement un aperçu général du contenu enseigné et évalué, respectivement. Sans vérifier le contenu des cours du NAIT ou avoir accès aux examens de la NACOR, il est difficile d'être certain du contenu exact couvert. Ainsi, nos méthodes pourraient être limitées par l'exactitude des renseignements contenus dans ces documents. Deuxièmement, notre analyse a été effectuée en fonction des 27 compétences que nous avons jugées nécessaires pour exécuter des examens de la réfraction et prescrire de manière sécuritaire et autonome. Toutefois, cette liste n'est pas exhaustive et toute analyse de ce type peut être sujette à la subjectivité, car différents professionnels de la vue peuvent considérer différentes compétences comme essentielles 
pour exécuter des EdR/P de manière sécuritaire. Enfin, une étude d'évaluation contrôlée aurait été idéale pour étudier les compétences et les connaissances des opticiens qui font des examens de la réfraction de l'Alberta. Au moment de la rédaction du présent document, il n'était pas possible de réaliser une évaluation contrôlée. Si, à l'avenir, le NAIT modifie son programme de cours et que la NACOR modifie son contenu d'examen pour couvrir toutes les compétences requises pour exécuter un EdR/P en toute sécurité, alors une étude d'évaluation contrôlée devrait être menée. Malgré ces limites, nous concluons que le programme d'études du NAIT et les compétences évaluées aux examens de la NACOR ne permettent pas aux opticiens d'acquérir les compétences appropriées pour exécuter un $\mathrm{EdR} / \mathrm{P}$ de façon autonome en toute sécurité. Nous espérons que les recherches futures pourront s'appuyer sur notre étude pour éclairer davantage cette importante question de recherche.

\section{CONCLUSION}

Cette étude visait à déterminer si les opticiens de l'Alberta qui font des examens de la réfraction possèdent les compétences appropriées pour exécuter ces examens et prescrire un appareil optique de façon autonome en toute sécurité. En analysant les compétences enseignées dans les programmes de sciences optiques du NAIT et les compétences évaluées dans les examens de la NACOR, nous avons conclu que les opticiens de l'Alberta qui exécutent des EdR ne possèdent pas à l'heure actuelle les compétences et les connaissances appropriées pour exécuter ces examens et prescrire un appareil optique de façon autonome en toute sécurité. Ainsi, en l'absence d'améliorations significatives des programmes de formation et d'évaluation actuellement en place, le fait d'accorder aux opticiens le pouvoir de prescrire mettrait la santé des Albertains en danger en autorisant les membres d'une profession qui ne possèdent pas les connaissances ou les compétences nécessaires pour garantir que le public ne soit pas soumis à un risque inutile à exécuter une activité restreinte. •

\section{AUTEUR-RESSOURCE \\ Dr. Gordon Hensel, OD. Email: registrar@collegeofoptometrists.ab.ca}

\section{RÉFÉRENCES}

1. Alberta College and Association of Opticians. 2019. What is an Optician? https://acao.ca/public/about-opticians/what-is-an-optician/. Accessed October 3, 2019.

2. Alberta College and Association of Opticians. 2018. Annual Council Report. https://acao.ca/wp-content/uploads/2018/05/Final-DraftCouncil-Reports.pdf. Accessed October 28, 2019.

3. Government of Alberta. 2019. Government Organization Act RSA 2000 Chapter G-10 Schedule 7.1. http://www.qp.alberta.ca/documents/Acts/g10.pdf. Accessed November 12, 2019.

4. College of Optometrists of Ontario. 2016. Optometric Practice Refer ence. https://www.collegeoptom.on.ca/images/pdfs/030_iD_COO_ OPR_book_WEB.pdf. Accessed September 25, 2019.

5. National Association of Canadian Optician Regulators. 2019. Programs Accredited by NACOR. http://www.nacor.ca/Programs_Accredited_By_NACOR.aspx. Accessed October 28, 2019.

6. Northern Alberta Institute of Technology. 2019. Optical Sciences - Eyeglasses. https://www.nait.ca/coned/optical-scienceseyeglasses?term=2020-winter. Accessed October 28, 2019.

7. Northern Alberta Institute of Technology. 2019. Optical Sciences - Contact Lenses. https://www.nait.ca/coned/optical-sciencescontact-lenses?term=2020-fall\&overviewtabs=program-overview. Accessed October 28, 2019.

8. Northern Alberta Institute of Technology. 2019. Optical Sciences Refraction. https://www.nait.ca/coned/optical-sciences-refraction. Accessed October 28, 2019.
9. National Association of Canadian Optician Regulators. 2019. Organization. http://www.nacor.ca/Organization.aspx. Accessed October 28, 2019.

10. Optometry Examining Board of Canada. About OEBC. http://www. oebc.ca/about. Accessed October 28, 2019.

11. National Association of Canadian Optician Regulators. 2011. Candidate's Examination Handbook. http://www.nacor.ca/my_folders/ For_Candidates/Candidate $\% 27 \mathrm{~s} \% 20$ Examination $\% 20$ Handbook. pdf. Accessed October 28, 2019.

12. Optometry Examining Board of Canada. 2019. Current OEBC Exam Blueprint. http://www.oebc.ca/clientuploads/OSCE/Blueprint Written_+_OSCE_ENG_(website).pdf. Accessed October 28, 2019.

13. Michaud L, Forcier P. Prevalence of asymptomatic ocular conditions in subjects with refractive-based symptoms. J. Optom 2014;7(3):153-160. https://doi.org/10.1016/j.optom.2013.08.003.

14. Alberta Health Services. and Alberta Innovates. Data Snapshot of Health and Healthcare Utilization in Alberta. 2013. https://public.tableau.com/views/AlbertaVisitRatesDiagnosisRatesandCosts/DiagnosisRates?\%3Aembed=y\&\%3Adispl ay_count=yes\&\%3AshowVizHome=no. Accessed March 2, 2020.

15. Northern Alberta Institute of Technology. 2019. OPSC695 - Refraction Clinical Course Overview. https://www.nait.ca/nait/continuing-education/courses/opsc695-refraction-clinical. Accessed October 28, 2019. 
Annexe A : Résultats d'apprentissage des cours de sciences optiques liés à la réfraction et à la prescription offerts par le NAIT

Tableau A1 : Couverture par le NAIT des compétences requises pour exécuter un EdR et prescrire en toute sécurité.

\begin{tabular}{|c|c|c|}
\hline $\begin{array}{l}\text { Compétences/ } \\
\text { capacités requises }\end{array}$ & $\underline{\text { Résultats d'apprentissage au NAIT }}$ & $\begin{array}{l}\text { Évalué dans } \\
\text { la pratique? }\end{array}$ \\
\hline Réfraction objective & $\begin{array}{l}\text { - Expliquer ce qu'est la réfraction objective, y compris le but d'un projecteur } \\
\text { et des lentilles d'essai, la rétinoscopie, le phoroptère et l'autophoroptère, et } \\
\text { l'ophtalmoscope } \\
\text { - Interpréter les résultats d'une réfraction objective } \\
\text { - Expliquer et analyser les résultats d'un EdR } \\
\text { - Utiliser l'autoréfractomètre et le rétinoscope } \\
\text { - Démontrer sa maîtrise d'un EdR complet avec l'analyse et l'interprétation des résultats } \\
\text { - Expliquer les résultats d'un EdR à un patient } \\
\text { - Déterminer les types de procédures servant à déterminer la réfraction, y compris } \\
\text { les procédures automatisées }\end{array}$ & Oui \\
\hline Réfraction subjective & $\begin{array}{l}\text { - Expliquer les principes et les procédures d'une détermination subjective de la } \\
\text { réfraction } \\
\text { - Analyser les résultats d'une réfraction subjective et résoudre les problèmes } \\
\text { - Utiliser le phoroptère } \\
\text { - Exécuter un examen de la réfraction subjective } \\
\text { - Démontrer sa maîtrise d'un EdR complet avec l'analyse et l'interprétation des } \\
\text { résultats } \\
\text { - Expliquer les résultats d'un EdR à un patient } \\
\text { - Déterminer les types de procédures servant à déterminer la réfraction, y compris } \\
\text { les procédures subjectives }\end{array}$ & Oui \\
\hline $\begin{array}{l}\text { Échelles d'acuité } \\
\text { à distance et à } \\
\text { proximité }\end{array}$ & $\begin{array}{l}\text { - Expliquer le dépistage des troubles de la vision, y compris l'acuité visuelle } \\
\text { - Effectuer un examen de l'acuité visuelle (à distance et à proximité, avec/sans } \\
\text { correction et test d'acuité au trou sténopéique) }\end{array}$ & Oui \\
\hline $\begin{array}{l}\text { Mesurer la courbure } \\
\text { cornéenne }\end{array}$ & $\begin{array}{l}\text { - Évaluer la santé de la cornée à l'aide d'instruments, y compris des lectures de } \\
\text { kératométrie et un topographe }\end{array}$ & Non \\
\hline $\begin{array}{l}\text { Mesurer la puissance } \\
\text { de la lentille à l'aide } \\
\text { d'un lensomètre }\end{array}$ & $\begin{array}{l}\text { - Analyser les paramètres à prendre en compte pour les lentilles unifocales et } \\
\text { multifocales, y compris la puissance d'une lentille à divers méridiens } \\
\text { - Interpréter une ordonnance de lentilles unifocales et multifocales, y compris la } \\
\text { puissance de la lentille } \\
\text { - Évaluer la tolérance aux lentilles cornéennes à l'aide d'un lensomètre } \\
\text { - Calculer la courbure et la puissance d'une lentille } \\
\text { - Mesurer la puissance des lentilles unifocales } \\
\text { - Mesurer la puissance des lentilles multifocales }\end{array}$ & Oui \\
\hline $\begin{array}{l}\text { Examen des } \\
\text { segments antérieur et } \\
\text { postérieur }\end{array}$ & $\begin{array}{l}\text { - Expliquer l'anatomie oculaire } \\
\text { - Expliquer la physiologie des yeux } \\
\text { - Reconnaître les pathologies oculaires dans la couche interne, la couche } \\
\text { intermédiaire, la couche externe, les cavités, la paupière et les muscles } \\
\text { extraoculaires } \\
\text { - Évaluer la santé oculaire et conseiller les patients } \\
\text { - Évaluer les pathologies oculaires de la paupière et la couche externe liées au port } \\
\text { de lentilles cornéennes } \\
\text { - Faire la distinction entre les maladies liées au port de lentilles cornéennes }\end{array}$ & Oui \\
\hline $\begin{array}{l}\text { Matériel de } \\
\text { photographie des } \\
\text { segments antérieur et } \\
\text { postérieur }\end{array}$ & - Aucun résultat d'apprentissage connexe & S.O. \\
\hline Tonométrie & - Aucun résultat d'apprentissage connexe & S.O. \\
\hline $\begin{array}{l}\text { Examen du champ } \\
\text { visuel informatisé }\end{array}$ & - Expliquer le dépistage des troubles de la vision, y compris le champ visuel & Non \\
\hline $\begin{array}{l}\text { Instrument à } \\
\text { balayage laser }\end{array}$ & - Aucun résultat d'apprentissage connexe & S.O. \\
\hline Lampe à fente & - Évaluer la santé de la cornée à l'aide d'une lampe à fente & Non \\
\hline
\end{tabular}




\begin{tabular}{|c|c|c|}
\hline $\begin{array}{l}\text { Compétences/ } \\
\text { capacités requises }\end{array}$ & Résultats d'apprentissage au NAIT & $\begin{array}{l}\text { Évalué dans } \\
\text { la pratique? }\end{array}$ \\
\hline Ophthalmoscope & - Expliquer la réfraction objective, y compris l'utilisation d'un ophtalmoscope & Non \\
\hline Lampe-stylo & - Aucun résultat d'apprentissage connexe & S.O. \\
\hline Pachymètre & - Aucun résultat d'apprentissage connexe & S.O. \\
\hline $\begin{array}{l}\text { Test de vision des } \\
\text { couleurs }\end{array}$ & $\begin{array}{l}\text { - Expliquer le dépistage des troubles de la vision, y compris la vision des couleurs } \\
\text { - Effectuer des tests de vision des couleurs }\end{array}$ & Oui \\
\hline $\begin{array}{l}\text { Détecter les signes de } \\
\text { maladies systémiques } \\
\text { et oculaires }\end{array}$ & $\begin{array}{l}\text { - Différencier les maladies systémiques, y compris les maladies cardiovasculaires, } \\
\text { endocriniennes, auto-immunes, infectieuses, systémiques et neurologiques } \\
\text { - Différencier les maladies qui ont une incidence sur le port de lentilles cornéennes, } \\
\text { y compris les maladies endocriniennes, auto-immunes et systémiques }\end{array}$ & Non \\
\hline Exophtalmomètre & - Aucun résultat d'apprentissage connexe & S.O. \\
\hline Sphygmomanomètre & - Aucun résultat d'apprentissage connexe & S.O. \\
\hline $\begin{array}{l}\text { Demander des tests } \\
\text { de laboratoire }\end{array}$ & - Aucun résultat d'apprentissage connexe & S.O. \\
\hline $\begin{array}{l}\text { Évaluer la fonction } \\
\text { accommodative }\end{array}$ & $\begin{array}{l}\text { - Effectuer des essais de vergence, y compris la fonction accommodative à l'aide de la } \\
\text { méthode du cylindre croisé } \\
\text { - Évaluer l'accommodation à l'aide de la méthode de Donders/«push-up test »/ } \\
\text { proximité et évaluer l'amplitude d'accommodation }\end{array}$ & Oui \\
\hline $\begin{array}{l}\text { Évaluer l'état de la } \\
\text { vision binoculaire }\end{array}$ & $\begin{array}{l}\text { - Expliquer les tests de vision binoculaire, y compris les trophies, les phories, } \\
\text { l'amblyopie et la vision binoculaire } \\
\text { - Effectuer des tests d'équilibre musculaire, y compris le test des reflets cornéens de } \\
\text { Hirschberg pour l'alignement des yeux, le test des reflets cornéens de Krimshy et le } \\
\text { test Worth Four-Dot. } \\
\text { - Évaluer les phories latérales et verticales à l'aide de prismes de Risley } \\
\text { - Effectuer des tests de stéréoacuité } \\
\text { - Exécuter un EdR subjective, y compris un test d'équilibre binoculaire avec prisme } \\
\text { de dissociation } \\
\text { - Expliquer le dépistage des troubles de la vision, y compris la motilité oculaire, la } \\
\text { vision binoculaire, la fonction pupillaire et les tests de couleur }\end{array}$ & Oui \\
\hline $\begin{array}{l}\text { Évaluer la motilité } \\
\text { oculaire }\end{array}$ & $\begin{array}{l}\text { - Expliquer la physiologie de l'œil, y compris les muscles extraoculaires } \\
\text { - Effectuer des tests d'équilibre musculaire }\end{array}$ & Oui \\
\hline $\begin{array}{l}\text { Évaluer la dominance } \\
\text { oculaire }\end{array}$ & - Aucun résultat d'apprentissage connexe & S.O. \\
\hline $\begin{array}{l}\text { Évaluer la } \\
\text { concomitance oculaire }\end{array}$ & - Aucun résultat d'apprentissage connexe & S.O. \\
\hline $\begin{array}{l}\text { Détecter les troubles } \\
\text { sensoriels/troubles } \\
\text { de la perception }\end{array}$ & - Aucun résultat d'apprentissage connexe & S.O. \\
\hline $\begin{array}{l}\text { Comprendre les } \\
\text { besoins des patients }\end{array}$ & $\begin{array}{l}\text { - Évaluer une prescription et déterminer si le patient a besoin d'un appareil optique } \\
\text { - Choisir une lentille unifocale appropriée pour un patient en analysant la } \\
\text { prescription et en déterminant les besoins du patient } \\
\text { - Choisir une lentille multifocale appropriée pour un patient en analysant la } \\
\text { prescription, en évaluant les besoins du patient et en choisissant le matériau et la } \\
\text { conception appropriés de la lentille }\end{array}$ & Oui \\
\hline $\begin{array}{l}\text { Communiquer les } \\
\text { options appropriées } \\
\text { au patient }\end{array}$ & $\begin{array}{l}\text { - Expliquer l'utilisation d'appareils spécialisés comme les lunettes de sécurité et de sport } \\
\text { - Adapter les techniques de communication et d'interaction pour améliorer la } \\
\text { communication avec les patients } \\
\text { - Conseiller les patients sur les appareils spécialisés à foyer simple, y compris les } \\
\text { lunettes de sécurité et les lunettes de sport }\end{array}$ & Oui \\
\hline
\end{tabular}


Annexe B : Compétences liées à I'EdR et à la prescription couvertes dans les examens de la NACOR et du BEOC.

Tableau B1: Couverture des compétences liées à l'EdR et à la prescription évaluées dans les examens de la NACOR et du BEOC.

\begin{tabular}{|c|c|c|}
\hline Compétences requises & $\begin{array}{l}\text { Couverture dans les examens de la } \\
\text { NACOR }\end{array}$ & Couverture dans les examens du BEOC \\
\hline Réfraction objective & - Non couvert & $\begin{array}{l}\text { Déterminer la réfraction objective par } \\
\text { rétinoscopie }\end{array}$ \\
\hline Réfraction subjective & - Non couvert & $\begin{array}{l}\text { - Déterminer la réfraction subjective à } \\
\text { distance à l'aide d'un phoroptère }\end{array}$ \\
\hline $\begin{array}{l}\text { Utiliser les échelles d'acuité à } \\
\text { distance et à proximité }\end{array}$ & $\begin{array}{l}\text { Effectuer un examen de suivi de } \\
\text { routine à six mois sur un modèle réel } \\
\text { (échelle d'acuité visuelle fournie) }\end{array}$ & $\begin{array}{l}\text { Déterminer l'acuité visuelle non } \\
\text { corrigée et avec correction binoculaire } \\
\text { et monoculaire à distance et à } \\
\text { proximité }\end{array}$ \\
\hline Mesurer la courbure cornéenne & $\begin{array}{l}\text { - Prendre les lectures K sur un œil d'un } \\
\text { modèle vivant }\end{array}$ & $\begin{array}{l}\text { Déterminer la courbure cornéenne à } \\
\text { l'aide de la kératométrie manuelle }\end{array}$ \\
\hline $\begin{array}{l}\text { Mesurer la puissance de la lentille à } \\
\text { l'aide d'un lensomètre }\end{array}$ & $\begin{array}{l}\text { - Calculer la «prescription compensée » } \\
\text { des lentilles à l'aide d'un lensomètre } \\
\text { - Mesurer le vertex des lentilles } \\
\text { cornéennes souples et des lentilles } \\
\text { perméables au gaz }\end{array}$ & $\begin{array}{l}\text { - Vérifier les paramètres des appareils } \\
\text { d'amélioration et de correction de la } \\
\text { vision }\end{array}$ \\
\hline $\begin{array}{l}\text { Examen des segments antérieur et } \\
\text { postérieur }\end{array}$ & - Non couvert & $\begin{array}{l}\text { Déterminer l'état des segments } \\
\text { antérieur et postérieur et de la santé } \\
\text { oculaire à l'aide de la biomicroscopie } \\
\text { du fond d'œil et de l'ophtalmoscopie } \\
\text { directe et indirecte }\end{array}$ \\
\hline $\begin{array}{l}\text { Matériel de photographie des } \\
\text { segments antérieur et postérieur }\end{array}$ & - Non couvert & $\begin{array}{l}\text { Déterminer les indications pour } \\
\text { l'imagerie du fond d'œil, la topographie } \\
\text { cornéenne, la tomographie par } \\
\text { cohérence optique, la tomographie } \\
\text { rétinienne de Heidelberg et } \\
\text { l'échographie B }\end{array}$ \\
\hline Pratiquer une tonométrie & - Non couvert & $\begin{array}{l}\text { - Déterminer la pression intraoculaire à } \\
\text { l'aide d'un tonomètre d'applanation }\end{array}$ \\
\hline $\begin{array}{l}\text { Effectuer un test informatisé du } \\
\text { champ visuel }\end{array}$ & - Non couvert & $\begin{array}{l}\text { - Déterminer les indications pour les } \\
\text { essais de périmétrie automatisés }\end{array}$ \\
\hline $\begin{array}{l}\text { Utiliser un instrument à balayage } \\
\text { laser }\end{array}$ & - Non couvert & $\begin{array}{l}\text { Déterminer les indications pour la } \\
\text { tomographie rétinienne de Heidelberg }\end{array}$ \\
\hline Utiliser une lampe à fente & $\begin{array}{l}\text { Exécuter, sur l'œil d'un modèle vivant, } \\
\text { un examen à la lampe à fente avec trois } \\
\text { des types d'éclairage suivants : type } \\
\text { "parallépipède », reflet spéculaire, } \\
\text { rétroillumination directe, diffusion } \\
\text { sclérotique et faisceau conique. }\end{array}$ & $\begin{array}{l}\text { Déterminer l'état de santé oculaire } \\
\text { antérieur et postérieur à l'aide de la } \\
\text { biomicroscopie }\end{array}$ \\
\hline Utiliser un ophtalmoscope & - Non couvert & $\begin{array}{l}\text { - Déterminer l'état des segments } \\
\text { antérieur et postérieur et l'état de santé } \\
\text { oculaire à l'aide de l'ophtalmoscopie } \\
\text { directe et indirecte }\end{array}$ \\
\hline Utiliser une lampe-stylo & $\begin{array}{l}\text { Mesurer la distance monoculaire et } \\
\text { l'écart pupillaire à l'aide d'une lampe- } \\
\text { stylo }\end{array}$ & - Non couvert \\
\hline Utiliser un pachymètre & - Non couvert & - Effectuer une pachymétrie \\
\hline
\end{tabular}




\begin{tabular}{|c|c|c|}
\hline Compétences requises & $\begin{array}{l}\text { Couverture dans les examens de la } \\
\text { NACOR }\end{array}$ & Couverture dans les examens du BEOC \\
\hline $\begin{array}{l}\text { Effectuer un test de vision des } \\
\text { couleurs }\end{array}$ & - Non couvert & $\begin{array}{l}\text { Déterminer l'état de la vision des } \\
\text { couleurs à l'aide des tests Ishihara, } \\
\text { D-15 ou Hardy Rand Ritter }\end{array}$ \\
\hline $\begin{array}{l}\text { Capacité de détecter les signes de } \\
\text { maladie systémique }\end{array}$ & $\begin{array}{l}\text { - Identifier diverses pathologies à partir } \\
\text { de photographies }\end{array}$ & $\begin{array}{l}\text { - Démontrer sa connaissance des signes } \\
\text { et des symptômes de problèmes } \\
\text { médicaux oculaires ou systémiques } \\
\text { nécessitant des soins immédiats }\end{array}$ \\
\hline Utiliser un exophthalmomètre & - Non couvert & - Non couvert \\
\hline Utiliser un sphygmomanomètre & - Non couvert & - Non couvert \\
\hline $\begin{array}{l}\text { Capacité de demander des tests de } \\
\text { laboratoire }\end{array}$ & - Non couvert & $\begin{array}{l}\text { Déterminer les indications pour les } \\
\text { analyses en laboratoire, y compris les } \\
\text { cultures, les analyses sanguines et } \\
\text { l'imagerie médicale }\end{array}$ \\
\hline $\begin{array}{l}\text { Capacité d'évaluer la fonction } \\
\text { d'accommodation }\end{array}$ & - Non couvert & $\begin{array}{l}\text { - Déterminer l'amplitude } \\
\text { d'accommodation à l'aide de la } \\
\text { technique «push-up » et de la } \\
\text { technique de Sheard }\end{array}$ \\
\hline $\begin{array}{l}\text { Capacité d'évaluer l'état de la vision } \\
\text { binoculaire }\end{array}$ & - Non couvert & $\begin{array}{l}\text { - Déterminer l'alignement oculaire à } \\
\text { l'aide de tests unilatéraux et à oeil } \\
\text { couvert en alternance } \\
\text { - Déterminer les réserves de vergence } \\
\text { à l'aide de barres de prisme ou de } \\
\text { prismes rotatifs } \\
\text { - Déterminer le point de convergence de } \\
\text { près } \\
\text { - Déterminer la phorie verticale à l'aide } \\
\text { de la tige et des prismes de Maddox }\end{array}$ \\
\hline $\begin{array}{l}\text { Capacité d'évaluer la motilité } \\
\text { oculaire }\end{array}$ & - Non couvert & $\begin{array}{l}\text { Évaluer la motilité oculaire à l'aide du } \\
\text { test H de Broad }\end{array}$ \\
\hline $\begin{array}{l}\text { Capacité d'évaluer la dominance } \\
\text { oculaire }\end{array}$ & - Non couvert & - Non couvert \\
\hline $\begin{array}{l}\text { Capacité d'évaluer la concomitance } \\
\text { des yeux }\end{array}$ & - Non couvert & $\begin{array}{l}\text { - Déterminer la concomitance à l'aide du } \\
\text { test à œil couvert en alternance }\end{array}$ \\
\hline $\begin{array}{l}\text { Capacité de détecter des désordres } \\
\text { sensoriels et d'autres problèmes de } \\
\text { perception }\end{array}$ & - Non couvert & $\begin{array}{l}\text { - Évaluer l'état sensoriel à l'aide d'un } \\
\text { test de stéréoacuité, d'un test de champ } \\
\text { visuel de confrontation, d'un test de } \\
\text { vision des couleurs, d'un test Worth-4- } \\
\text { Dot et d'un test de Pelli-Robson }\end{array}$ \\
\hline $\begin{array}{l}\text { Capacité de comprendre les besoins } \\
\text { des patients }\end{array}$ & - Non couvert & $\begin{array}{l}\text { - Déterminer les valeurs et les préférences } \\
\text { du patient en matière de soins }\end{array}$ \\
\hline $\begin{array}{l}\text { Capacité de communiquer des } \\
\text { options pour satisfaire aux } \\
\text { exigences professionnelles et de } \\
\text { loisirs des patients }\end{array}$ & - Non couvert & $\begin{array}{l}\text { - Fournir des conseils sur la sécurité } \\
\text { oculaire en milieu de travail et lors } \\
\text { d'activités récréatives }\end{array}$ \\
\hline
\end{tabular}




\section{Annexe C : Diagnostics liés à l'œil et coûts des soins de santé en Alberta}

Tableau C1 : Diagnostics liés à l'œil et coûts en Alberta

\begin{tabular}{|l|c|c|}
\hline Type de diagnostic & $\begin{array}{l}\text { Nombre de diagnostics (AF } \\
\text { 2013/2014-2015/2016) }\end{array}$ & $\begin{array}{l}\text { Coût (en millions de dollars, AF } \\
\text { 2014/2015) }\end{array}$ \\
\hline Diagnostics de problèmes oculaires chroniques & 204461 & 15 \\
\hline Diagnostics de problèmes oculaires aigus & 13895 & 14 \\
\hline Conjonctivite et autres inflammations oculaires & 53201 & 28 \\
\hline Glaucome & 97738 & 84 \\
\hline Cataracte & 91887 & 1,3 \\
\hline Amblyopie & 3330 & 1,3 \\
\hline Cécité/perte de vision & 6609 & 13 \\
\hline Dégénérescence maculaire & 15945 & 239,6 \\
\hline Total & 487066 & \\
\hline
\end{tabular}

Les données ont été tirées du document Data Snapshot of Health and Healthcare Utilization in Alberta des Services de santé de l'Alberta ${ }^{14}$. Le coût associé à chaque diagnostic comprend les coûts liés à l'hôpital (hospitalisation, urgence et clinique) et la facturation directe des médecins. Le nombre de diagnostics comprend tous les diagnostics posés entre les exercices $2013-$ 2014 et 2015-2016. Une personne peut avoir reçu plus d'un diagnostic au cours de cette période de trois ans. 